\title{
The Half-metallic Properties of (001) and (110) Surfaces of CsSe from the First-principles
}

\author{
Beata Bialek and Jae Il Lee* \\ Department of Physics, Inha University, Incheon 22212, Republic of Korea
}

(Received 8 January 2016, Received in final form 16 February 2016, Accepted 18 February 2016)

\begin{abstract}
We investigated the half-metallicity and magnetism at the (001) and (110) surfaces of CsSe in cesium chloride and zinc-blende structures by using the all-electron full-potential linearized augmented plane wave method within the generalized gradient approximation. From the calculated local density of states, we found that all the surfaces preserve the half-metallicity of the bulk structures. The surfaces with a greater polarity have stronger ferromagnetic properties when terminated with Se atoms; the non-polar surfaces do not change their electronic or magnetic properties considerably as compared with the bulk structures.
\end{abstract}

Keywords : half-metal, surface magnetism, first-principles method

\section{Introduction}

There has been considerable interest in designing new materials for application in spin transport electronics. One of the basic processes in devices for spintronics is spinpolarized electron injection through a heterojunction. The process is the most efficient when the spin polarization of electrons is nearly a hundred percent. Half-metals, materials with a total electron spin polarization at the Fermi level, are therefore very desirable candidates for the applications. Half-metallicity was first discovered in the early $80 \mathrm{~s}$ of the previous century by de Groot et al. who studied magnetic properties of a half-Heusler alloy $\mathrm{NiMnSb}$ [1]. Since then, a general trend in searching for half-metals was to investigate the properties of ternary compounds of similar structure, i.e., half- or full-Heusler. In the compounds containing elements from transition metal series the presence of a sp element was important for stabilizing ferromagnetic half-metallicity [2]. After reporting a discovery of $\mathrm{d}^{0}$ ferromagnetism in an insulating sp material [3], ferromagnetic half-metallicity was predicted in many compounds without a transition metal in their structure [4-10]. Promising candidates for spintronics were searched for not only by altering a chemical composition, but also by investigating various crystal structures.

CThe Korean Magnetics Society. All rights reserved.

*Corresponding author: Tel: +82-32-860-7654

Fax:+82-32-872-7562, e-mail: jilee@inha.ac.kr
As for the composition, the materials exhibiting sp ferromagnetic half-metallicity became desirable because of a small magnetic moment, which reduces the demagnetizing fields and assure a small energy loss. As for the crystal structure, not only the most energetically stable compounds were investigated, but also those with the structure commensurate with the most common structures of semiconducting materials, such as zinc blende and rock-salt structures.

Recently, half-metallic ferromagnetism was predicted in CsSe compound by Karaca et al. [11]. It was found that the most stable ferromagnetic ground state of that $\mathrm{sp}$ material is with cesium chloride crystal structure. Other structures, i.e., zinc blende, rock-salt, nickel arsenide, and wurtzite, were also investigated and were found to have ferromagnetic half-metallic properties, as well.

There is a long way from designing a material to its possible application in electronic devices. In the process of designing, most often perfectly periodic compounds are investigated, while it is a well-known fact that a break in periodicity may considerably change the properties of a perfect crystal. Additionally, processes important for a performance of electronic devices occur at surfaces. Computational studies of the electronic and the magnetic properties of surfaces are often challenging, but our group succeeded in studies of surfaces of many materials reported as half-metals in their bulk structures [12-14]. According to our results, half-metallic properties of a bulk structure, even if robust to unit cell volume changes, do 
not guarantee that surfaces of the material retain the properties of the bulk. Predicting how the properties of surfaces are different (or similar) to the properties of the underlying bulk structure may shorten the path to successful application of the investigated materials in future electronic devices.

In this paper we discuss the half-metallic properties of (001) and (110) surfaces of CsSe in cesium chloride $(\mathrm{CsCl})$ and zinc blende $(\mathrm{ZnS})$ structures calculated with the use of a first-principles method.

\section{Computational Details}

According to Karaca et al. [11], CsSe is a ferromagnetic half-metal in $\mathrm{CsCl}$, rock-salt, $\mathrm{ZnS}$, nickel arsenide, and wurtzite-type structures. The most stable energetically is CsCl-type CsSe; for this structure $4.362 \AA$ equilibrium lattice constant was calculated. The total energy of the ZnS-type CsSe was about $0.425 \mathrm{eV}$ higher than that of the CsCl-type, and was defined by $8.221 \AA$ lattice constant in equilibrium. ZnS-type structure is an important one for application in spintronics, for heterostructures of ferromagnetic half-metals and semiconductors are frequent parts of electronic devices. In both the $\mathrm{CsCl}$ and $\mathrm{ZnS}$-type structures of $\mathrm{CsSe}$, two surfaces may have quite different properties: (001) surface is a polar surface with two possible terminations, i.e., consisted of either $\mathrm{Cs}$ or $\mathrm{Se}$ atoms at the topmost layer, while (110) surface is a nonpolar one terminated with both Cs and Se atoms. We use the reported lattice constants to model the above mentioned surfaces by nine-layer slabs. The slabs were thick enough for the four atomic layers on both sides of the center layer to provide enough screening for the center of the slab, which can be assumed to mirror the bulk properties of the CsSe. The distances between the atomic layers were $2.055 \AA$ and $2.907 \AA$ for (001) and (110) surfaces in the ZnS-type CsSe, respectively. In the CsCl-type CsSe the distances between the layers were $2.181 \AA$ and $3.084 \AA$ for (001) and (110) surfaces, respectively. Since the CsSe bulk in CsCl-type structure retains its half-metallic properties in a wide range of lattice constants [11] we assumed that small relaxation and reconstruction cannot affect much the electronic properties of the system. Also, we assumed that similar behavior is true for the ZnS-type structure. Therefore, we calculated the electronic properties of unrelaxed and unreconstructed surfaces only.

In order to resolve the electronic structure of the CsSe surfaces, the Kohn-Sham equation [15] was solved selfconsistently in terms of the full-potential linearized augmented plane wave (FLAPW) method [16, 17], within generalized gradient approximation (GGA) of PerdewBurke-Ernzerhof type [18] to the exchange-correlation potential.

Lattice harmonics with $l \leq 8$ were employed to expand the charge density, the potential, and the wave functions inside the muffin-tin radii of 2.50 a.u. for $\mathrm{Cs}$ and $\mathrm{Se}$ atoms. The number of basis functions was about 200 per atom. Integration was performed over a $13 \times 13$ mesh of kpoints inside the irreducible two dimensional Brillouin zone (BZ) for each system. All core electrons were treated fully relativistically, while valence states were treated scalar relativistically, without spin-orbit coupling. Self-consistency was assumed when the difference between input and output charge densities was less than $1 \times 10^{-4}$ electrons/(a.u. $)^{3}$.

\section{Results and Discussion}

Fig. 1 shows the calculated density of states (DOS) projected on atoms in the (001) Se-terminated surface of the CsCl-type CsSe compound. The features of the atoms from the center, next to the center, the subsurface, and the surface layers are shown. The layers are denoted as C, S$3, \mathrm{~S}-1$, and S, respectively. The numbers included in the panels denote the calculated partial magnetic moments on the atoms. In each panel the Fermi level is set to zero. The minority electron states are plotted in negative scale. It is clear that the (001) Se-terminated surface of the CsCl-type CsSe retains the half-metallic properties of the bulk. This is confirmed also by the calculated value of the total magnetic moment of the system, which is $6.00 \mu_{\mathrm{B}}$. In the bulk CsCl-type CsSe, energy band gap of $3.75 \mathrm{eV}$ was predicted for majority (spin-up) electrons [4]. Considering that the center layer of the (001) CsCl-type CsSe surface
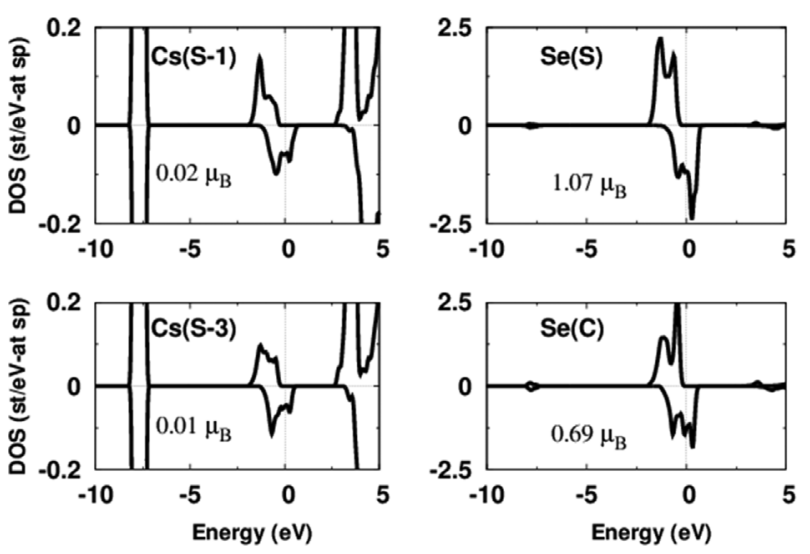

Fig. 1. Spin-polarized atom-projected density of states calculated for the Se-terminated (001) CsCl-type CsSe. The Fermi level is set to zero. The numbers inside the panels denote the calculated magnetic moments on the atoms in the slab. 
mirrors the properties of the bulk we estimate that the energy band gap width is about $3.40 \mathrm{eV}$, which is close to the calculated value for the bulk structure. Due to surface effects, the energy gap width decreases slightly to 3.00 $\mathrm{eV}$. Interestingly, the decrease in the energy gap between valence and conduction band in majority electron channel is accompanied by a small widening of a half-metallic energy gap, which is defined as relative energy of the top of the majority electron valence band with respect to the Fermi level and which determines the smallest energy necessary for electron excitation from the majority valence band to the Fermi level.

The partial magnetic moments calculated for the bulklike atoms in the surface, $0.69 \mu_{\mathrm{B}}$ for $\operatorname{Se}(\mathrm{C})$ and $0.01 \mu_{\mathrm{B}}$ for $\mathrm{Cs}(\mathrm{S}-3)$, are in perfect agreement with those reported by Karaca et al. [11]. The effect of broken periodicity is noticeable at the top and subsurface layers: The calculated magnetic moment on $\mathrm{Se}(\mathrm{S})$ atom increases to $1.07 \mu_{\mathrm{B}}$, and the magnetic moment on $\mathrm{Cs}(\mathrm{S}-1)$ atom increases slightly to $0.02 \mu_{\mathrm{B}}$. The distribution of electrons within Cs atoms muffin tins does not change much with their position in the slab. On the other hand, majority l-like charges on Se atoms at the top layer are larger than those in the center layer, and there is a considerable decrease in the magnitude of minority l-like charges within muffin tin of $\mathrm{Se}(\mathrm{S})$ compared with $\mathrm{Se}(\mathrm{C})$. In result, the number of the occupied spin-down states in the top layer decreases, and the polarization of electrons increases.

The calculated total magnetic moment of the Csterminated (001) CsSe surface of $\mathrm{CsCl}$ structure is also integer $(3.00 \mu \mathrm{B})$. However, compared with the Seterminated (001) surface of CsCl-type CsSe, Cs-terminated one may not be a very stable half-metallic system. As it is seen from the partial DOS plots shown in Fig. 2, the halfmetallic energy gap, though still present, is very narrow, approximately $0.10 \mathrm{eV}$. Such a different effect may be explained by much stronger delocalization of Cs s electrons at the surface; the number of occupied states contributed by $\operatorname{Cs}(\mathrm{S})$ atom is much lower than that contributed by $\mathrm{Cs}(\mathrm{C})$ atom. The electrons of $\mathrm{Se}(\mathrm{S}-1)$ atoms also become more delocalized, and in result the magnetic moments on atoms at the top and subsurface layers are decreasing. Se atoms are much more sensitive to the surface effects; the magnetic moment on $\mathrm{Se}(\mathrm{S}-1)$ atom is only half of what it is in the bulk.

Although CsCl-type CsSe appears to be the most stable energetically, ZnS-type CsSe may be more desirable as a material for application in spintronics. According to the results of our calculations, both Se-terminated and Csterminated (001) surfaces of ZnS-type CsSe retain their ferromagnetic half-metallic properties of the bulk. As it is
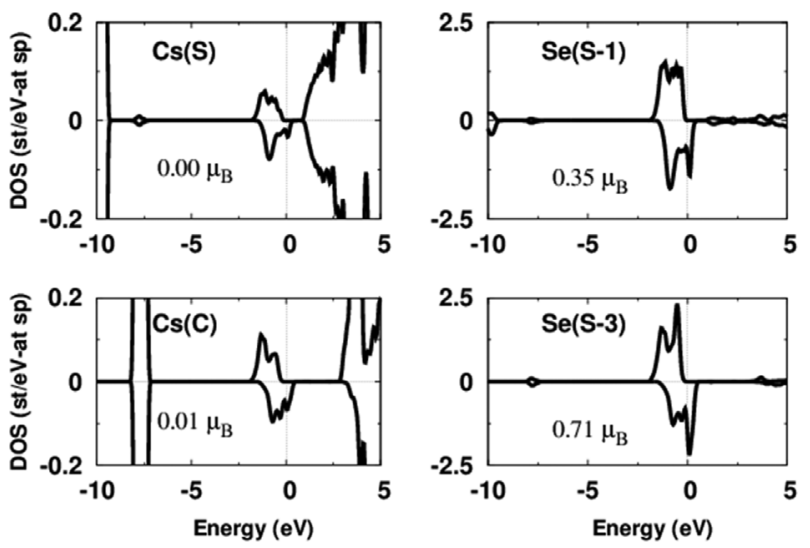

Fig. 2. Spin-polarized atom-projected density of states calculated for the Cs-terminated (001) CsCl-type CsSe. The Fermi level is set to zero. The numbers inside the panels denote the calculated magnetic moments on the atoms in the slab.
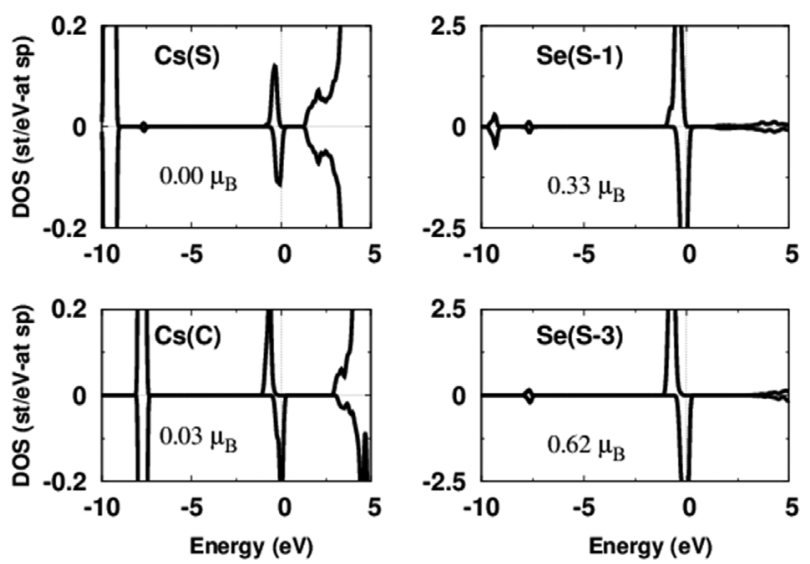

Fig. 3. Spin-polarized atom-projected density of states calculated for the Cs-terminated (001) ZnS-type CsSe. The Fermi level is set to zero. The numbers inside the panels denote the calculated magnetic moments on the atoms in the slab.

the case in CsCl-type (001) surfaces of the compound, there are differences between the Se-terminated and the Cs-terminated surfaces: due to the surface effects, the energy gap in the majority (spin-up) electron channel is considerably decreased from the bulk value to $1.36 \mathrm{eV}$ and the width of the half metallic energy gap is as small as $0.3 \mathrm{eV}$. This effect is due to a large delocalization of $\mathrm{s}$ electron of $\operatorname{Cs}(\mathrm{S})$ atoms, which is clearly seen in Fig. 3. The fact that the states near the Fermi level, contributed by $\mathrm{Se}(\mathrm{S}-1)$ electrons, are not much different from the states in the bulk indicates efficient screening of the subsurface layers by the Cs atoms at the top layer of the slab.

In Fig. 4 the partial DOS plots are shown for the Seterminated (001) surface of ZnS-type CsSe. In this system, the magnitudes of both the energy gap in the majority 

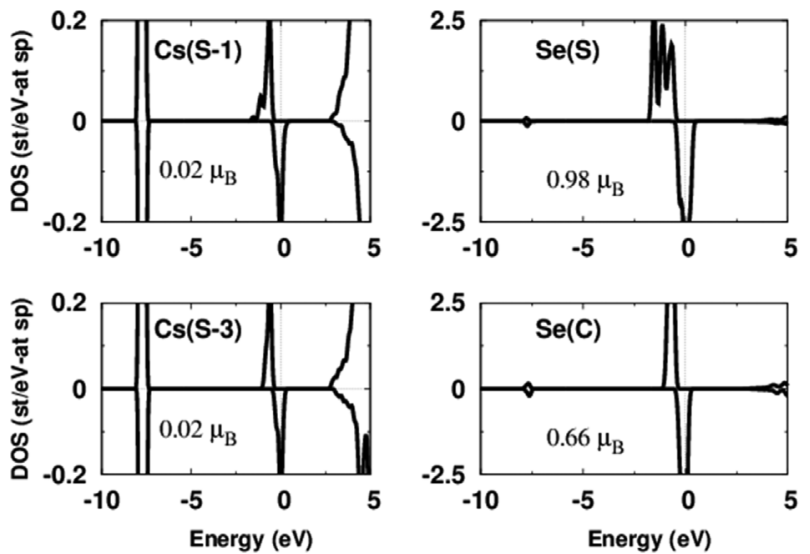

Fig. 4. Spin-polarized atom-projected density of states calculated for the Se-terminated (001) ZnS-type CsSe. The Fermi level is set to zero. The numbers inside the panels denote the calculated magnetic moments on the atoms in the slab.

electron channel and the half-metallic energy gap do not change much. The magnetic moment of $\mathrm{Cs}(\mathrm{S}-1)$ atom is the same as the bulk value, and the spin polarization on $\mathrm{Se}(\mathrm{S})$ atom is increased to $0.98 \mu_{\mathrm{B}}$ from $0.66 \mu_{\mathrm{B}}$ on the bulk-line $\mathrm{Se}(\mathrm{C})$ atom.

Compared with CsCl-type (001) surface of the CsSe compound the electron states near the Fermi energy are much more strongly localized, which is explained by a different arrangement of the atoms in the two slabs. In the ZnS-type structure, the distance between Cs and Se atoms is $3.08 \AA$, while in CsCl-type structure it is $3.78 \AA$. The greater distance allows for distribution of electrons into interstitial region in a larger degree than in ZnS-type structure.

In cubic structures, (110) surfaces are more densely packed and less open than (001) surfaces. Additionaly, in case of binary compounds, there is only one termination possible. In both CsCl-type and ZnS-type, each layer of a slab cut in $<110\rangle$ direction consists of the two types atoms, and so the surfaces are non-polar. Therefore, we expected some differences between the electronic and the magnetic properties of the (001) and the (110) surfaces of CsSe.

In Fig. 5 and in Fig. 6 the plots of the partial DOS calculated for (110) surfaces of CsCl-type and ZnS-type $\mathrm{CsSe}$ are shown, and the magnitudes of the magnetic moments on the atoms are indicated.

Both CsCl-type and ZnS-type (110) surfaces of the CsSe compound are half-metallic. Due to a larger in-plane distance between the atoms in the CsCl-type surface, the electronic bands are wider than those in the ZnS-type surface.

However, in contrary to what we found for the (001)
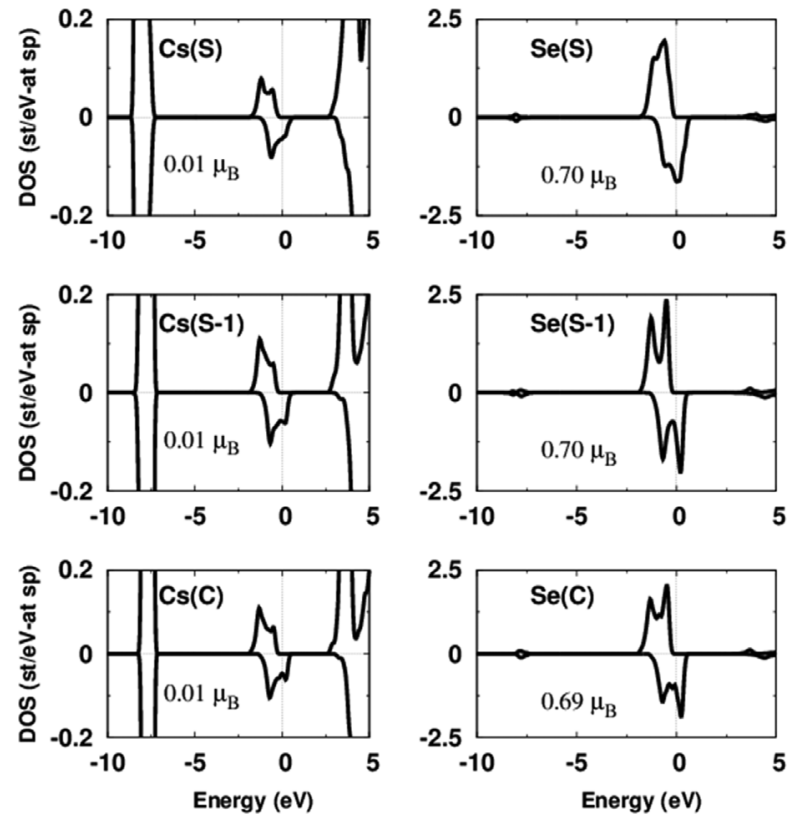

Fig. 5. Spin-polarized atom-projected density of states calculated for the (110) CsCl-type CsSe. The Fermi level is set to zero. The numbers inside the panels denote the calculated magnetic moments on the atoms in the slab.
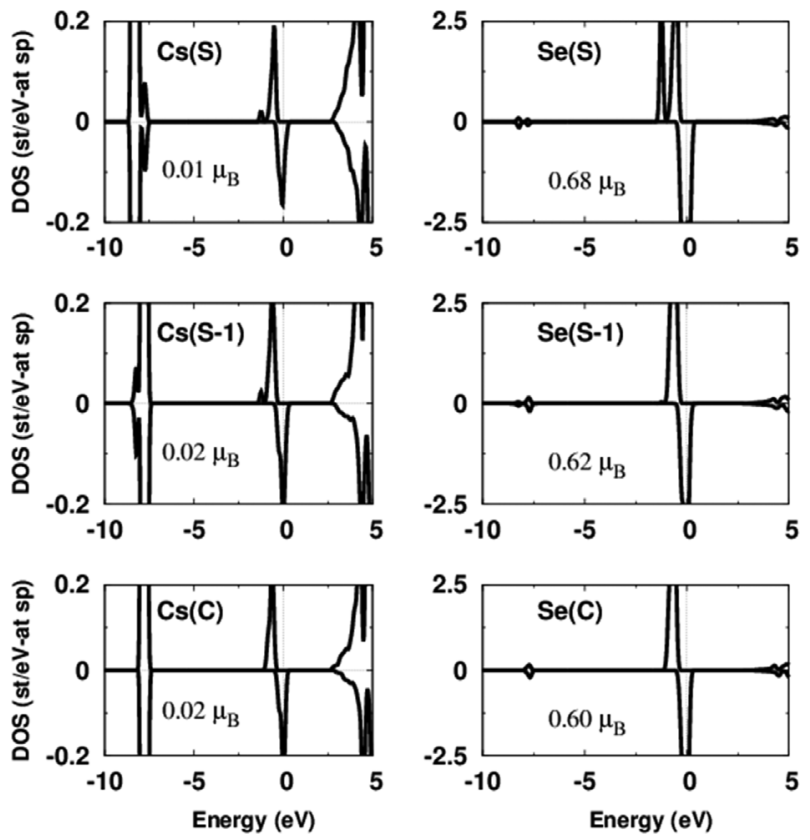

Fig. 6. Spin-polarized atom-projected density of states calculated for the (110) ZnS-type CsSe. The Fermi level is set to zero. The numbers inside the panels denote the calculated magnetic moments on the atoms in the slab.

surface, the magnetic properties of the atoms in the topmost and the subsurface layers of (110) surfaces do not change considerably compared with the bulk properties. 
Especially in the CsCl-type (110) surface there is hardly any quantitative effect on the magnetic properties of the atoms. The effect is slightly larger in the ZnS-type (110) surface, in which the calculated magnetic moment changes from $0.60 \mu_{\mathrm{B}}$ on $\mathrm{Se}(\mathrm{C})$ atom to $0.68 \mu_{\mathrm{B}}$ on $\mathrm{Se}(\mathrm{S})$ atom.

\section{Conclusion}

We investigated the surface effect on the half-metallic properties of CsSe ferromagnetic half-metal in CsCl-type and $\mathrm{ZnS}$-type crystal structures. In all the investigated surfaces, i.e., (001) and (110), the half-metallicity was retained. We found that the (001) surfaces are more sensitive to the surface effects, especially when terminated with Cs atoms. The most noticeable changes in the electronic structure were observed in the Cs-terminated CsCl-type (001) surface; the energy gap in the minority electron channel decreased considerably. In both the CsCl-type and the ZnS-type (001) surfaces terminated by Cs atoms the Se atoms of subsurface layers became demagnetized by $0.36 \mu_{\mathrm{B}}$ and $0.29 \mu_{\mathrm{B}}$, respectively. On the contrary, the magnetization of the topmost layer of Se-terminated surfaces increased in both the CsCl-type and the ZnS-type (001) surfaces. In the (110) surfaces the electronic and the magnetic properties of the surfaces remained quite similar to the properties of the bulk. That may indicate that the in-plane interactions are more important for shaping the properties of the (110) surfaces than are the out-of-plane effects.

\section{Acknowledgements}

This work was supported by the Inha University Research Fund.

\section{References}

[1] R. A. de Groot, F. M. Mueller, P. G. van Engen, and K. H. J. Bushow, Phys. Rev. Lett. 50, 2024 (1983).

[2] Van A. Dinh, K. Sato, and H. K. Yoshida, J. Comput. Theor. Nanos. 62589 (2009).

[3] M. Venkatesan, C. B. Fitzgerald, and J. M. D. Coey, Nature 430, 630 (2004).

[4] G. Y. Gao, K. L. Yao, E. Şaşıŏglu, L. M. Sandratskii, Z. L. Liu, and J. L. Jiang, Phys. Rev. B 75, 174442 (2007).

[5] G. Y. Gao and K. L. Yao, J. Appl. Phys. 106, 053703 (2009).

[6] G. Y. Gao and K. L. Yao, Appl. Phys. Lett. 91, 082512 (2007).

[7] Q. S. Shao and H. Zhao, J. Supercond. Nov. Magn. 25, 2063 (2012).

[8] G. Y. Gao, K. L. Yao; Z. L. Liu, J. L. Jiang, L. H. Yu, and Y. L. Shi, J. Phys.: Condens. Matt. 19, 315222 (2007).

[9] H. Rozale, A. Lakdja, A. Amar, A. Chahed, and O. Benhelal, Comp. Mater. Sci. 69, 229 (2013).

[10] J. Chen, G. Y. Gao, K. L. Lao, and M. H. Song, J. Alloys Compd. 509, 10172 (2011).

[11] M. Karaca, S. Kervan, and N. Kervan, J. Alloys Compd. 639, 162 (2015).

[12] B. Bialek and J. I. Lee, J. Magn. 20, 1 (2015).

[13] B. Bialek, J. I. Lee, and M. Kim, Comp. Mater. Sci. 81, 510 (2014).

[14] B. Bialek and J. I. Lee, J. Magn. 18, 375 (2013).

[15] W. Kohn and L. J. Sham, Phys. Rev. A 140, 1133 (1965).

[16] E. Wimmer, H. Krakauer, M. Weinert, and A. J. Freeman, Phys. Rev. B 24, 6864 (1981), and references therein.

[17] M. Weinert, E. Wimmer, and A. J. Freeman, Phys. Rev. B 26, 4571 (1982).

[18] J. P. Perdew, K. Burke, and M. Ernzerhof, Phys. Rev. Lett. 77, 3865 (1996). 